\title{
New service proposed for expectant mothers after Motherisk closure
}

\author{
— Cite as: CMAJ 2019 June 3;191:E619-20. doi: 10.1503/cmaj.109-5755
}

Posted on cmajnews.com on May 14, 2019.

$\mathbf{T}$ he Society of Obstetricians and Gynaecologists of Canada (SOGC) is hoping to create a resource similar to Motherisk, the program recently shut down by The Hospital for Sick Children (SickKids) in Toronto. Motherisk provided information on the safety of medications and substances during pregnancy. The service received about 200 calls a day; $20 \%$ were from health care providers and $80 \%$ from pregnant or breastfeeding women or their family members, according to Dr. David Naylor, the former interim president and CEO of SickKids.

Many health providers and parents expressed dismay about the closure. But according to Dr. Navindra Persaud, a family physician and scientist at St. Michael's Hospital, Motherisk provided biased information about the effectiveness or safety of some medications. He's worried that SOGC isn't an appropriate body to take over the resource and questions whether it's needed at all.

In a public statement, SickKids cited lack of funding as the reason for the closure, in part because of "adverse publicity arising from concerns about the quality of work carried out by a hair analysis laboratory that also carried the Motherisk name." A report by Justice Susan Lang, prompted by a Toronto Star investigation, found that Motherisk's hair tests were flawed and unreliable. A second Star investigation raised concerns over research by former Motherisk director, Dr. Gideon Koren, including failures to disclose funding from pharmaceutical companies such as Duchesnay, maker of the anti-nausea drug Diclectin (doxylamine-pyridoxine) .
But SickKids maintains that the hotlines weren't compromised. In an email, Naylor wrote that the Motherisk helplines relied on "broader literature and research."

Persaud noted, however, that in "the case of Diclectin, Dr. Koren was involved in much of the research that was done, so it doesn't seem to be the case that they were relying on the broader literature there." He also suggested that Motherisk may have overemphasized the safety or effectiveness of other drugs, including antidepressants.

He argued that referring pregnant patients to Motherisk "was just an easy thing we [doctors] could do rather than looking up the information and assuming responsibility for that discussion on the benefits and risks of drugs and pregnancy."

But according to Dr. Jennifer Blake, CEO of the SOGC, Motherisk filled a vital role. "A place where all of the literature is assessed and compiled is essential," she said. Given that drugs taken in pregnancy can affect the genetics of the fetus and future generations, "the stakes are high." A fetus's susceptibility to a drug can also change substantially depending on the dosage or stage of development. Understanding and communicating

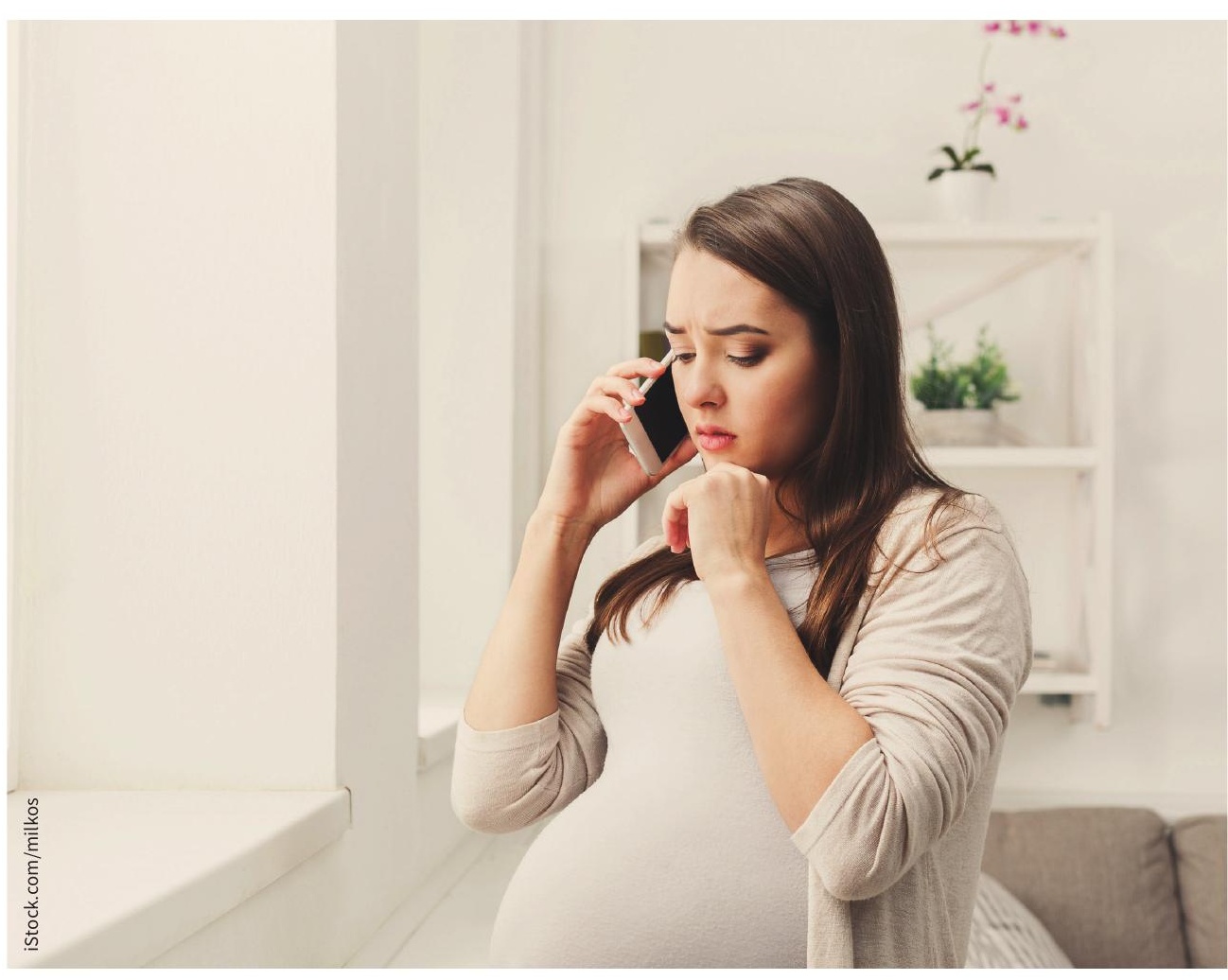

The Motherisk program received about 200 phone calls a day. 
these complexities is "beyond what a doctor has time to do, amongst all the other things they're trying to do."

Blake said the society is working to connect funders and experts to create "a national network" that would research drugs and substances in pregnancy and provide information to health providers and the public, much like Motherisk did.

Christina Chambers, program director of the US-based resource MotherToBaby, said her program is monitoring the increase in calls from Canada, because if the volume of calls that went to Motherisk were redirected to MotherToBaby, "that would be impossible for us to absorb."

For most callers, reassurance that the glass of wine they had in early pregnancy or the medication they're on is safe is "the takeaway message," said Chambers. "That is hard to measure, the benefit of the reduction in anxiety." She pointed out that women using substances may be more comfortable calling a confidential hotline than disclosing it to a doctor.

Blake said that although phone calls may still be necessary, a searchable, online database that would allow a patient or provider to enter information on the dosage and stage of pregnancy "might be more appropriate."

Persaud questions whether the SOGC should lead the resource, considering that the society has received funding from Duchesnay. He's concerned that the SOGC continues to recommend doxylamine-pyridoxine despite his 2018 reanalysis showing the trial used to prove the drug's effectiveness was flawed. And according to a 2017 commentary in Canadian Family Physician, "there is not, at this time, clear evidence that the combination of doxylaminepyridoxine is more effective in the management of [nausea and vomiting in pregnancy] than pyridoxine alone."

Blake maintains that the SOGC's ethical guidelines ensure that funding by pharmaceutical companies doesn't affect its recommendations. "I don't have an opinion as to what went on at Motherisk," she said, "but I think for anything that's done going forward, there needs to be strong oversight and clear guidelines to avoid any real, potential or perceived conflicts of interest."

Disclaimer: Dr. Navindra Persaud is a CMAJ associate editor; his views do not necessarily reflect those of CMAJ. He was not involved in the editorial decisionmaking for this article.

Wendy Glauser, Toronto, Ont. 